EVOLUTION

\section{Memories of mammoths}

\author{
If elephants never forget, the \\ memaries of mammoths need \\ a little prompting. Nevertheless, \\ inventive approaches to the \\ extraction and sequencing of \\ DNA from mammoths preserved \\ in Siberian perma frost are allowing \\ direct access to the deeper \\ memories of elephant evolution. \\ There has been much debate \\ about whether the woolly \\ marnmoth (Mammuthus \\ primigenius) - that archetype \\ of everything icy and Palaeolithic \\ - was more closely related to the \\ extant African or Asian elephant \\ (Loxodonta africanus and Elephas \\ maximus, respectively). Analysis of \\ the complete mitochandrial genome \\ of a mammoth by Hofreiter and \\ colleagues (Nature 439, 724-727; \\ 2006) provides the answer: \\ mammoths are more closely related
}

to Asian elephants, but only just. Current wisdom hasit that the lineages leading to mammoths and both extantelephantspecies diverged about 6 million years ago in Africa. The new data suggest that the Afric an lineage split first, followed around 440,000 years later by the separation between Asianelephants and mammoths. Now that the phylogenetic relationships are slightly clearer, it would be interesting to discover how other extinct elephants fitinto the picture. One thinks of elephants such as

Anancus and

Palaeoloxodon that

foraged in temperate Europe during the Pleistocene- not to mention the mighty Mammuthus trogontherii, which at up to five metres tall was possibly the largest species of elephant ever, making the woolly mammoth look, if not dwarfed, then at least somewhatpetite.

These questions may not be answered using ancientDNA, however. The permafrost environment seems to favour the preservation of ancient DNA inquantity, from mammoths as

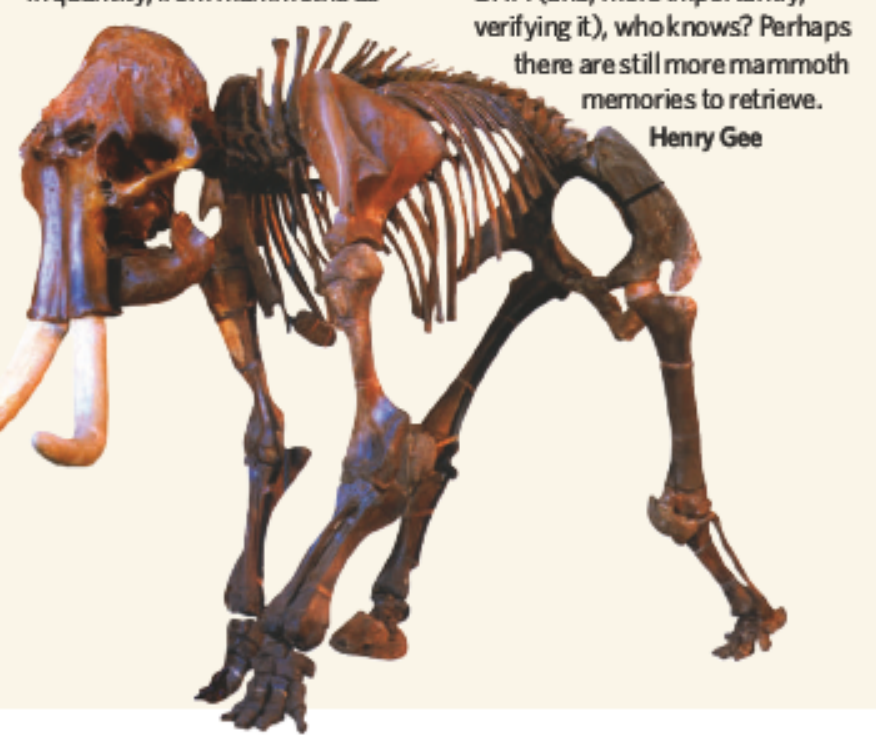

well as other species, as shown by Poinar and colleagues (Science 311,392-394; 2006). But the likelihood of finding sufficiently informative DNA from species living outside the Arctic is almost certainly very much less. Yet, given the advances in sequencing ancient DNA (and, more importantly, rifying it), whoknows? Perhaps scanning tunnelling microscopy and modelling to show that this conventional analysis cannot fully explain electron transport in nanoscale silicon films. Using silicon-onoxide membranes, already employed as the basis of low-capacitance, fast-switching devices, they demonstrate that transport is feasible even with very thin membranes, and for doping levels at which the $\mathrm{Si}-\mathrm{SiO}_{2}$ interface effectively depletes all carriers within the semiconductor. Electronic conduction is seemingly, in this case, not controlled by dopants in the bulk of the solid.

So what does control electron transport in silicon membranes? The authors provide compelling arguments to implicate surface states that come about when the single dangling bond of two neighbouring silicon surface atoms combine to form so-called $2 \times 1$ dimers. This process results in filled and empty surface states that are very close in energy to the valence band of the bulk silicon, and, in the author' model, generate holes that dominate charge transport (Fig. 1).

This behaviour is probably not unique to the $2 \times 1$ surface configuration, or even to silicon. In fact, any surface 'termination' that provides energy levels that are low enough to accept electrons from the valence band should suffice. In the case of silicon, this would obviate the need to work with the notoriously reactive $2 \times 1$ surface, which is impractical for any real device application. That would open up new avenues to the manufacture of nanoscale devices.

The surface states associated with the $2 \times 1$ surface can be eliminated by chemical

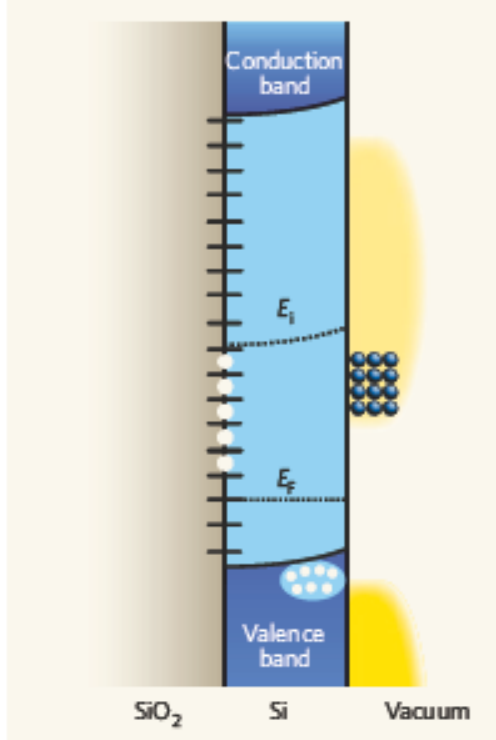

Figure 1| The band structure of a silicon-oninsulator membrane. Trap states (short lines) at the silicon-silicon-dioxide interface in Zhang and colleagues' thin silicon membrane ${ }^{1}$ depress the Fermi energy $\left(E_{\mathrm{F}}\right)$ of the membrane to below its intrinsic level $\left(E_{1}\right)$, hindering conduction. But surface effects create allowed energy states (yellow) just above the valence band, into which electrons (blue circles) can be excited thermally, producing 'holes' (white circles) that can propagate through the valence band. This surface effect in silicon works equivalently to the doping effect in bulk silicon in enhancing the material's conduction properties. (Adapted from ref. 1.) reaction: terminations such as hydrogen or halogens would suppress the surface states and prevent the generation of holes within the film. Such terminations could be patterned, providing a method of creating locally conducting and insulating regions of the substrate. But generating new surface states close to the energy gap is best achieved by tailoring the gap between the highest occupied and lowest unoccupied orbitals of molecular species attached to the surface. This would in principle allow the generation of both hole- and electron-conducting regions in the film.

Although such surface-state models provide a potentially powerful route to control the electronic structure of nanoscale semiconductors, further studies will be needed to allow a detailed understanding of the transport mechanisms that result. Temperaturedependent conductivity measurements and high-resolution scanning tunnelling spectroscopy would be useful in this regard. Mechanism aside, Zhang and colleagues demonstrate $^{1}$ that, in the quest for ever-smaller electronic devices, surprises and opportunities abound.

John J. Boland is in the Centre for Research on Adaptive Nanostructures and Nanodevices (CRANN) and the School of Chemistry, Trinity College Dublin, Dublin 2, Ireland. e-mail:jboland@tcd.ie

1. Zhang, P. et al. Nature 439, 708-706 (2006)

2. Erwin, S.et al Nature 436,91-94 (2005).

3. Shinada, T_ Okamoto, S_Kobayashi, T. \& Ohdomari, L Nature 437, 1128-4131 (2005).

4. Hamers, R.J.etal. Acc. Chem. Res. 33 617-624 (2000) 